\title{
Mechanical properties of sisal fibre reinforced urea- formaldehyde resin composites
}

\author{
J. B. Zhong, J. Lv, C. Wei \\ Key Laboratory of New Processing Technology for Nonferrous Metals and Materials, Ministry of Education, Guilin \\ University of Technology, Guilin541004, China
}

Received 6 June 2007; accepted in revised form 12 August 2007

\begin{abstract}
Alkali-treated sisal fibres were used as novel reinforcement to obtain composites with self-synthesized ureaformaldehyde resin as matrix phase. The composites were prepared by means of compression molding, and then the effects of sisal loading on mechanical properties such as impact strength, flexural strength, and wear resistance were investigated. In addition, water uptake was studied and structural features were revealed by the scanning electron microscopy (SEM). The composite with $30 \mathrm{wt} \%$ sisal fibres gives excellent flexural strength, water absorption, and especially the wear resistance showing that it has the most superior bonding and adhesion of all the composites. In particular, the highest value $9.42 \mathrm{~kJ} / \mathrm{m}^{2}$ of charpy impact strength is observed in the composite with $50 \mathrm{wt} \%$ sisal fibre. SEM micrographs of impact fractured and worn surfaces clearly demonstrate the interfacial adhesion between fibre and matrix. This work shows the potential of sisal fibre (SF) to improve the composite wear resistance and to be used in fibreboard.
\end{abstract}

Keywords: polymer composites, mechanical properties, sisal fibres, urea-formaldehyde resin, micro-structure

\section{Introduction}

Fibre reinforced polymer composites are being used in almost every type of applications in our daily life and its usage continues to grow at an impressive rate. The manufacture, use and removal of traditional composite structures usually made of glass, carbon and aramid fibres are considered critically because of the growing environmental consciousness [1]. In recent years, there is a growing interest in the use of biofibres as reinforcing components for thermoplastics and thermosets. Sisal fiber (SF), a member of the Agavaceae family is a biodegradable and environmental friendly crop. Moreover, sisal is a strong, stable and versatile material and it has been recognized as an important source of fibre for composites [2-4].

It is generally accepted that the mechanical properties of fiber reinforced polymer composites are

*Corresponding author, e-mail: glweichun@glite.edu.cn

(C) BME-PT and GTE controlled by factors such as nature of matrix, fiber-matrix interface, fiber volume or weight fraction, fiber aspect ratio etc. Many scientists are working in this field and the reinforcement of polymer with SF has been widely reported. Low-density polyethylene-sisal [5], Polyester-sisal [6], epoxysisal [7], polypropylene-sisal [8, 9], urea-formaldehyde-sisal [10], phenol-formaldehyde-sisal [11, 12], polyvinyl-acetate-sisal [13], and starch-basedsisal [14] are some of the promising systems. A further attempt to use sisal fibre as reinforcement for heat and pressure applications has been found in the literature [15]. Manikandan Naira et al. [16] studied the thermal behaviour of polystyrene composites reinforced with short SF by means of thermogravimetric and dynamic mechanical thermal analysis. It has been found [17] that matrix cracking, fibre bridging, fibre breakage and pull-out are the major fracture modes of sisal textile reinforced 
composites with pre-cracks under the static loading condition. However, despite the fact that several methods have been used and great strides have been made, there is still some lack of knowledge about wear resistance property of sisal reinforced polymer composites.

Urea-formaldehyde resins (UFR) are the most prominent examples of the class of thermosetting resins usually referred to as amino resins, even though new research efforts are needed to address to offset its major disadvantage. The use of UFR as a major adhesive by the forest products industry is due to a number of advantages, including low cost, ease of use under a wide variety of curing conditions, low cure temperatures, water solubility, resistance to microorganisms and to abrasion, hardness, excellent thermal properties etc.

The present paper describes the changes in the Charpy impact, flexural and wear resistance properties of SF/UFR blend composite as a function of weight fraction of SF. In addition to this, the effect of SF loading on water absorption tendencies of the composites has also been examined. Meanwhile, the surface morphology is studied and described. We conduct this study to determine whether the compatibility between UFR and SF is strong or weak, and to evaluate the abrasive wear resistance of the composites. It is anticipated that this study may open the way for future investigations in the use of SF in fiberboard so that the range of sisal's potential applications can be widened.

\section{Experimental}

\subsection{Materials}

Fibres were soaked in $2 \% \mathrm{NaOH}$ solution in a water bath where the temperature was maintained throughout at $22 \pm 2^{\circ} \mathrm{C}$ for $24 \mathrm{~h}$, then washed with distilled water and left to dry at room temperature before being put in an oven for $15 \mathrm{~h}$ at $70^{\circ} \mathrm{C}$. Finally, fibers were chopped into $2-3 \mathrm{~mm}$ size.

Liquid urea-formaldehyde resin was synthesized by us. The ratio of urea to formaldehyde used was 1:1.4. Melamine was added for improving adhesion strength and its amount was $8 \mathrm{wt} \%$ of urea. The reaction temperature was $90 \pm 1^{\circ} \mathrm{C}$ and reaction time was about $2.5 \mathrm{~h}$. It had an average viscosity of $3.5 \cdot 10^{-2}-4.5 \cdot 10^{-2} \mathrm{~Pa} \cdot \mathrm{s}$, $\mathrm{pH}$ between 7.0 and 7.5 , $50 \pm 1 \%$ solid content and $<1 \%$ free formaldehyde content.

\subsection{Sample preparation}

A mixture of SF, UFR, and inorganic filler $\mathrm{CaCO}_{3}$, curing agent $\left(\mathrm{NH}_{4} \mathrm{Cl}, 3 \mathrm{wt} \%\right.$ of resin) and mold release agent (zinc stearate, $1 \mathrm{wt} \%$ of the total mixture mass) was prepared, and then put it in an oven for $2 \mathrm{~h}$ at $90^{\circ} \mathrm{C}$. The blends were taken for composite fabrication available as such. The specimens were produced through compression molding under a pressure of $8 \mathrm{MPa}$ at $140-145^{\circ} \mathrm{C}$ for $4 \mathrm{~min}$. After that, the composites were post-cured at $120^{\circ} \mathrm{C}$ for $2 \mathrm{~h}$. The composites containing 30, 40,50, 60 and $70 \mathrm{wt} \%$ SF, contained UFR 50, 40, 30, 20 and $10 \mathrm{wt} \%$, respectively.

\subsection{Characterization}

Charpy impact strengths of the specimens (120x $10 \times 4 \mathrm{~mm}$ length, width and depth, respectively) were determined with an impact tester (Chengteh China, Model JC-25 4J) in the flatwise, unnotched mode. The striking velocity was $2.9 \mathrm{~m} / \mathrm{s}$ and the reported values reflect an average from five measurements.

Three-point bend tests were performed using Shimadzu electronic universal test machine (Model AG-201).The specimens were $120 \times 10 \times 4 \mathrm{~mm}$ length, width and depth, respectively. The span of the supports was $60 \mathrm{~mm}$, and the loading speed was $2 \mathrm{~mm} / \mathrm{min}$. The reported values reflect an average from five measurements.

Sliding wear tests were conducted on an abrasive wear tester (Yihua China, Model M-2000) at room temperature according to GB3960-83. The diameter of the steel ring (45\#steel) was $40 \mathrm{~mm}$, the hardness was 45-50 HRC, and the rotation speed of the steel was $200 \mathrm{rpm}$ during operation. Before the testing, the specimen and the steel ring were washed with acetone and air dried. The size of the wear specimens were $30 \times 7 \times 6 \mathrm{~mm}$ length, width and depth, respectively. The tests were performed at a normal load of $200 \mathrm{~N}$ and for $40 \mathrm{~min}$. The wear volume loss and friction coefficient were adopted to evaluate wear resistance property. The reported values reflect an average from five measurements.

Water absorption testing of composites has been carried out by taking circular sheet which the diameter was $50 \mathrm{~mm}$ and thickness was $4 \mathrm{~mm}$.Samples were first dried by heating in an oven at $50^{\circ} \mathrm{C}$ for about $24 \mathrm{~h}$, weighed $\left(W_{1}\right)$ and then soaked in distilled water in beakers at room temperature. After 

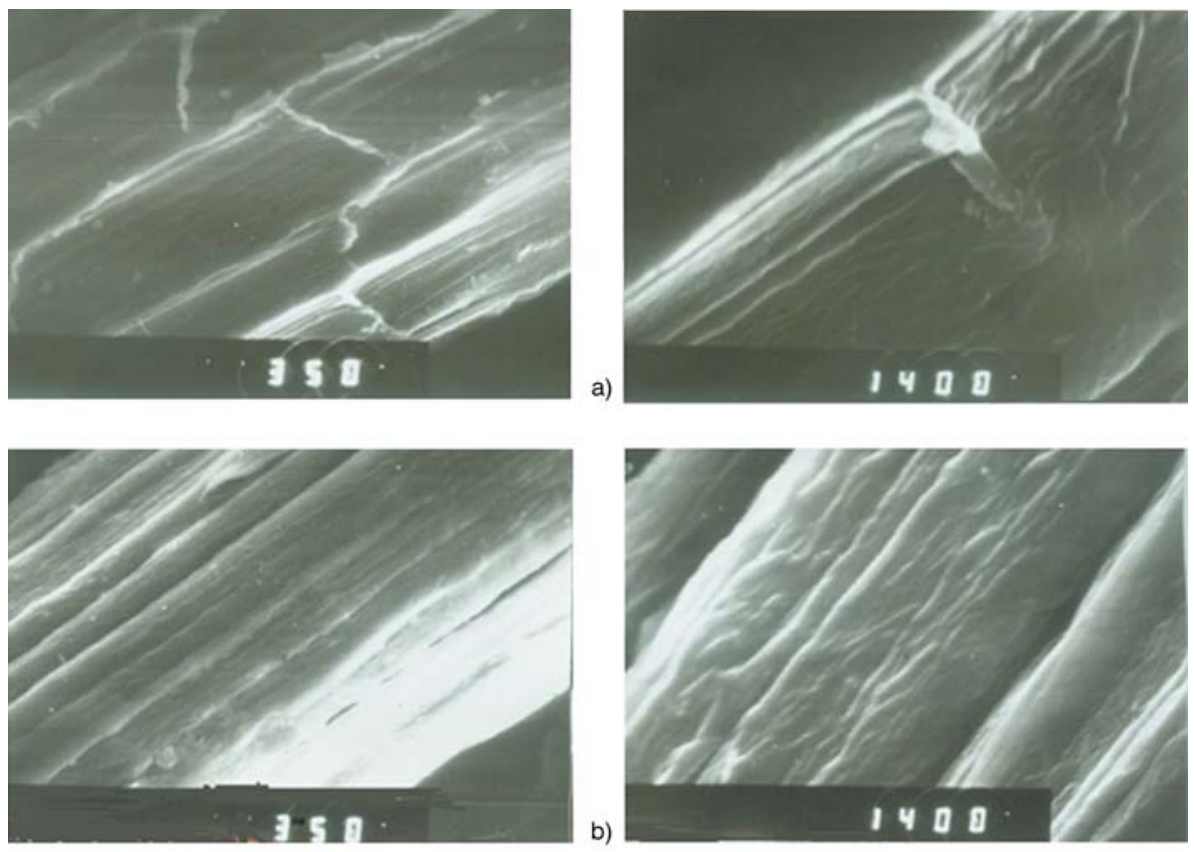

Figure 1. SEM micrographs of (a) untreated SF, (b) alkali-treated SF

$24 \mathrm{~h}$, the composite samples were removed from water, dried by a cotton cloth and weighed $\left(W_{2}\right)$. Percentage water absorption of the samples was calculated. The reported values reflect an average from five measurements.

SEM micrographs of SF surfaces and fractured surfaces following impact were taken using a scanning electron microscope (Model JEOL JSM-6380LV). Prior to SEM evaluation, the samples were coated with carbon by sputtering technique. The worn surfaces were observed using the same method.

\section{Results and discussion}

\subsection{SEM examination of treated SF}

SEM micrographs of untreated and alkali-treated $\mathrm{SF}$ are shown in Figure 1. It can be observed that the untreated SF (a) presents a network structure and includes waxes and other low molar mass impurities. Compared to the (b), it is clear that SF gets thinner after treatment. It is possible that treatment led to micro-fiber fibrillation. The surfaces of the SF (b) become rather smoother as compared to that of untreated SF. In addition, this fibrillation could have increased the effective surface area available for contact with the matrix in the composites, as well as reduced the diameter of sisal fibers, thereby increasing their aspect ratio. This may offer better fiber-matrix interface adhesion and improve stress transfer. These will give rise to improvement in mechanical properties.

\subsection{Impact strength}

Impact strength is defined as the ability of a material to resist the fracture under stress applied at high speed. The impact properties of composite materials are directly related to its overall toughness. Composite fracture toughness is affected by interlaminar and interfacial strength parameters [18]. Figure 2 shows the variation of Charpy unnotched impact strength of SF/UFR composite with SF loading. The impact strength of composite with $30 \mathrm{wt} \% \mathrm{SF}$ is $5.78 \mathrm{~kJ} / \mathrm{m}^{2}$.With increase in SF load-

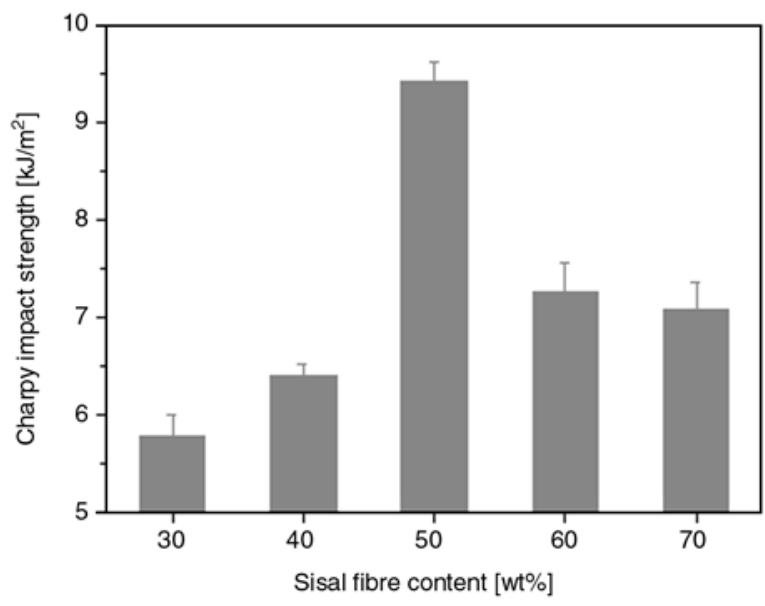

Figure 2. Effects of sisal fibre loading on the impact strength of SF/UFR composites 
ing from 30 to $50 \mathrm{wt} \%$, the impact strength increases by $62.98 \%$, namely achieves $9.42 \mathrm{~kJ} / \mathrm{m}^{2}$. With further increase in SF loading (60 wt \%), there is a considerable decrease in impact strength. The impact strength of a composite is influenced by many factors including the matrix fracture, fibrematrix debonding and fibre pull out. It has been reported by van der Oever et al. [19] that the Charpy impact strength decreases with enhanced fibre-matrix adhesion. For the composite with $30 \mathrm{wt} \% \mathrm{SF}$, the UFR is sufficient and excessive; when compared to other samples. This is likely to cause a drop in toughness and can explain why the impact strength is much lower. Moreover, the superior strength of composite with $50 \mathrm{wt} \%$ may be associated with proper interfacial adhesion between the fibre and matrix; the fibres have reasonable amounts and act as stress transferring medium. In addition, the interspaces and stress concentrations could have acted as crack initiation points during impact. Therefore, inferior impact strength is obtained in the composite containing $60 \mathrm{wt} \% \mathrm{SF}$.
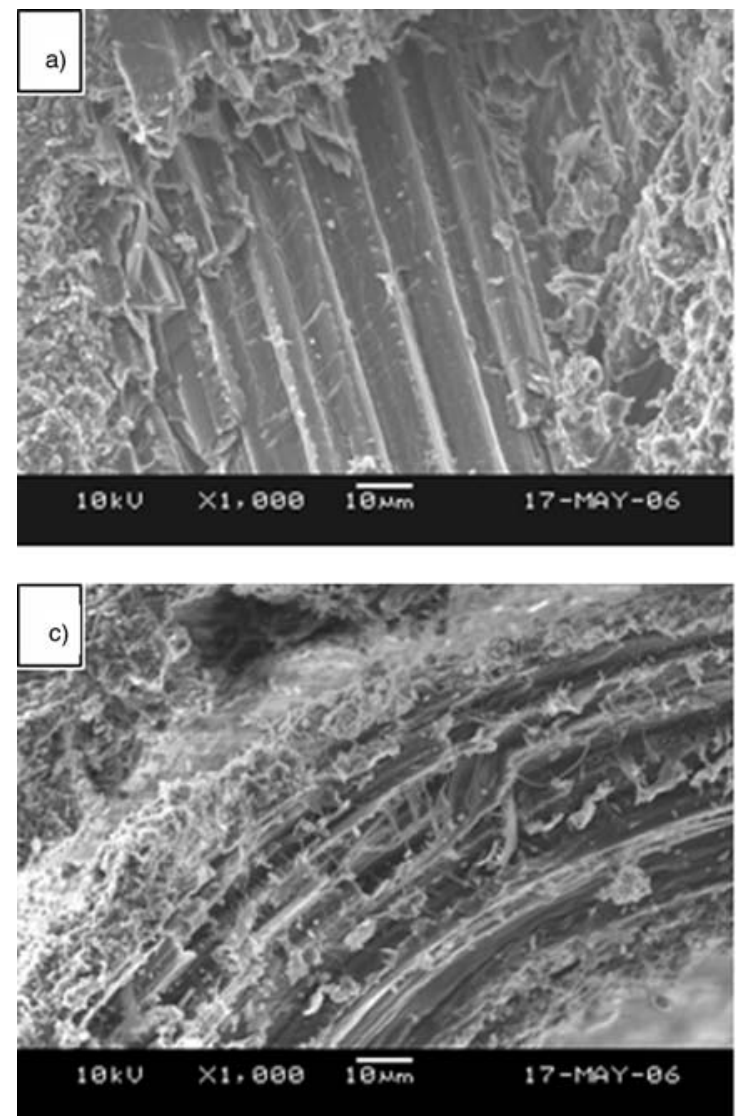
hence, the interfacial friction stress and chemical bond between matrix and fibre is generally higher shoot up with the increase of the SF. These fibers

Figure 3 shows several micrographs of the fractured surface of SF/UFR composite on impact. The images (a) and (b) show the fibres are well anchored in the matrix and a very small amount of fibres are pulled out. The fractured surfaces are smooth and clear. This is observed due to the brittle nature of the matrix. It can be concluded that the fracture mainly happens in the matrix and the performance of fibres is not available. However, in (c) and (d), fiber bundle debonding, deformation in the fiber leading to microcracking and fracture, and fibers splitting are presented. Toughness is significantly enhanced due to the fibres acting as stress transferring medium.

\subsection{Flexural property}

Results from the bend tests on SF/UFR composite are given in Table 1. It is observed that with decrease of SF content from 70 to $30 \mathrm{wt} \%$, the flexural strength increases sharply i. e. from 15.28 to $58.58 \mathrm{MPa}$, increasing by about $283.4 \%$. Meanwhile, the flexural modulus increases from 1.59 to $7.63 \mathrm{GPa}$. As the improved mixing will provide
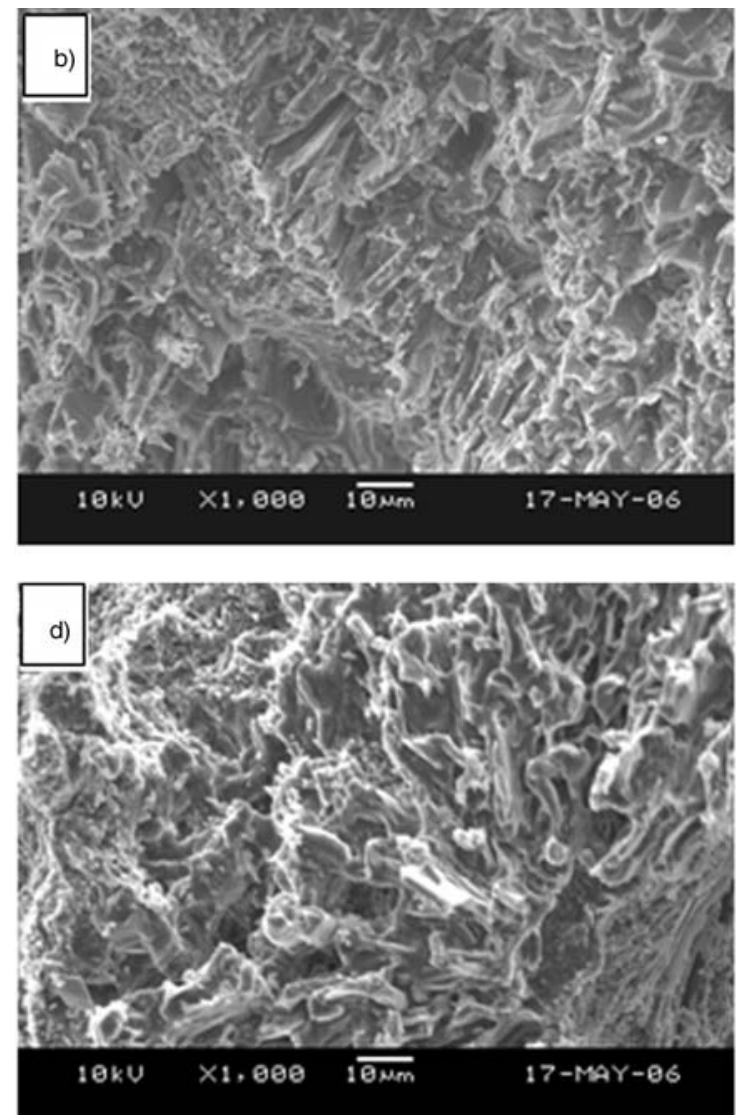

Figure 3. SEM micrograph of Charpy impact fractured surface of SF/UFR composites with (a, b) 30 wt $\%$ and (c, d) $50 \mathrm{wt} \%$ sisal fibre 
Table 1. Effect of sisal fibre loading on the bending property of SF/UF composite

\begin{tabular}{|c|c|c|c|c|c|}
\hline $\begin{array}{c}\text { SF content } \\
{[\mathbf{w t} \%]}\end{array}$ & $\begin{array}{c}\text { Flexural strength } \\
{[\mathbf{M P a}]}\end{array}$ & $\begin{array}{c}\text { Flexural modulus } \\
{[\mathbf{G P a}]}\end{array}$ & $\begin{array}{c}\text { Density } \\
{\left[\mathbf{g} / \mathbf{c m}^{\mathbf{3}}\right]}\end{array}$ & $\begin{array}{c}\text { Specific strength } \\
{\left[\mathbf{M P a} \cdot \mathbf{c m}^{\mathbf{3} / \mathbf{g}]}\right.}\end{array}$ & $\begin{array}{c}\text { Specific modulus } \\
{\left[\mathbf{G P a} \cdot \mathbf{c m}^{\mathbf{3}} \mathbf{\mathbf { g }}\right]}\end{array}$ \\
\hline 30 & 58.58 & 7.63 & 1.53 & 38.29 & 4.99 \\
\hline 40 & 55.80 & 5.27 & 1.52 & 36.71 & 3.47 \\
\hline 50 & 53.07 & 4.93 & 1.48 & 35.86 & 3.33 \\
\hline 60 & 37.73 & 4.09 & 1.44 & 26.20 & 2.84 \\
\hline 70 & 15.28 & 1.59 & 1.22 & 12.52 & 1.31 \\
\hline
\end{tabular}

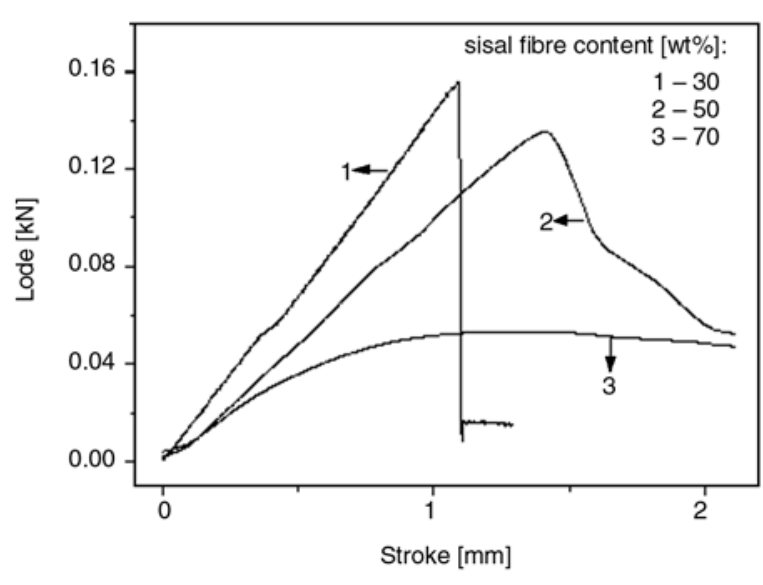

Figure 4. Relationships between lode and stroke as a function of sisal fibre loading

better distribution of sisal, the bridging gaps between the fibres can conduct more effectively. To obtain qualitative information about the flexural behavior of the composite, the load-stroke traces are taken into consideration. In Figure 4, although the composite with $30 \mathrm{wt} \%$ sisal loading yields catastrophically, it bears a higher load than other samples, resulting in a higher flexural strength. In addition, it deforms less until maximum load, which gives a higher flexural modulus. As a consequence, the flexural strength and the flexural modulus of the composites show larger values. Apparently, the stroke of the composite with $50 \mathrm{wt} \%$ sisal loading is larger, and this can support the impact results as discussed earlier.

\subsection{Wear resistance property}

The variation of wear volume loss and friction coefficient with weight fraction of SF for steadystate sliding of SF/UFR composite against the stainless steel ring under dry conditions is shown in Figures 5 and 6. Evidently the wear volume losses and friction coefficients of SF/UFR composite go up with increasing sisal weight fraction. Better performance of the sample with $30 \mathrm{wt} \%$ sisal relies

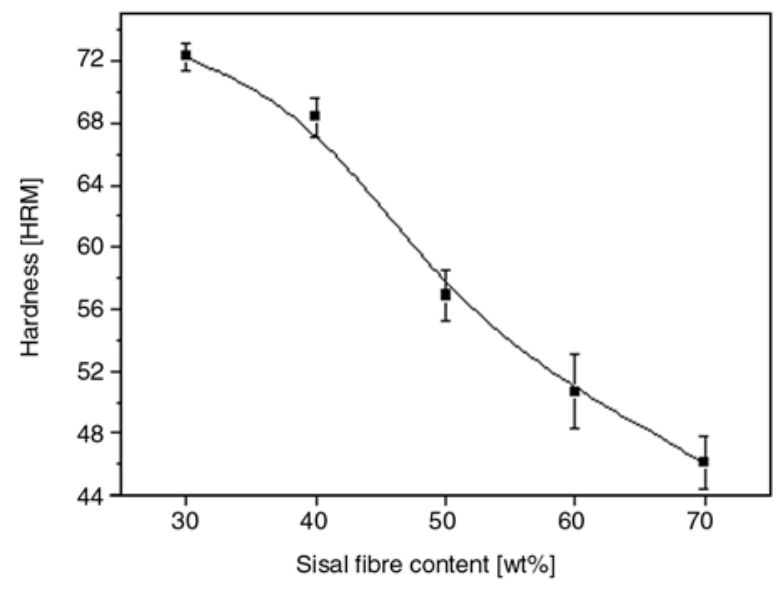

Figure 5. Effects of sisal fibre loading on the hardness of SF/UFR composites

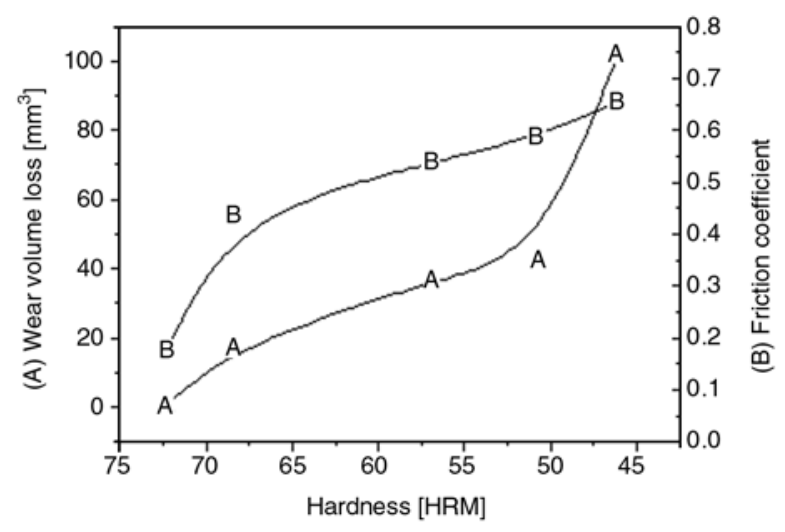

Figure 6. Relationships of the wear volume loss and friction coefficient to the hardness of SF/UFR composites

heavily on its strong interfaces, and the higher surface hardness nature of the matrix. This indicates that wear of the composite is mostly due to the fibre-matrix adhesion and partly due to the nature of components.

The surface in Figure 7a is significantly smoother and one can conclude that the inclusion of SF hinders the microcutting, microploughing and deformation of the worn surface. Thus, adhesion and ploughing of the composite are much reduced, leading to a very low wear rate. In addition, the resin may be released from the composite during 

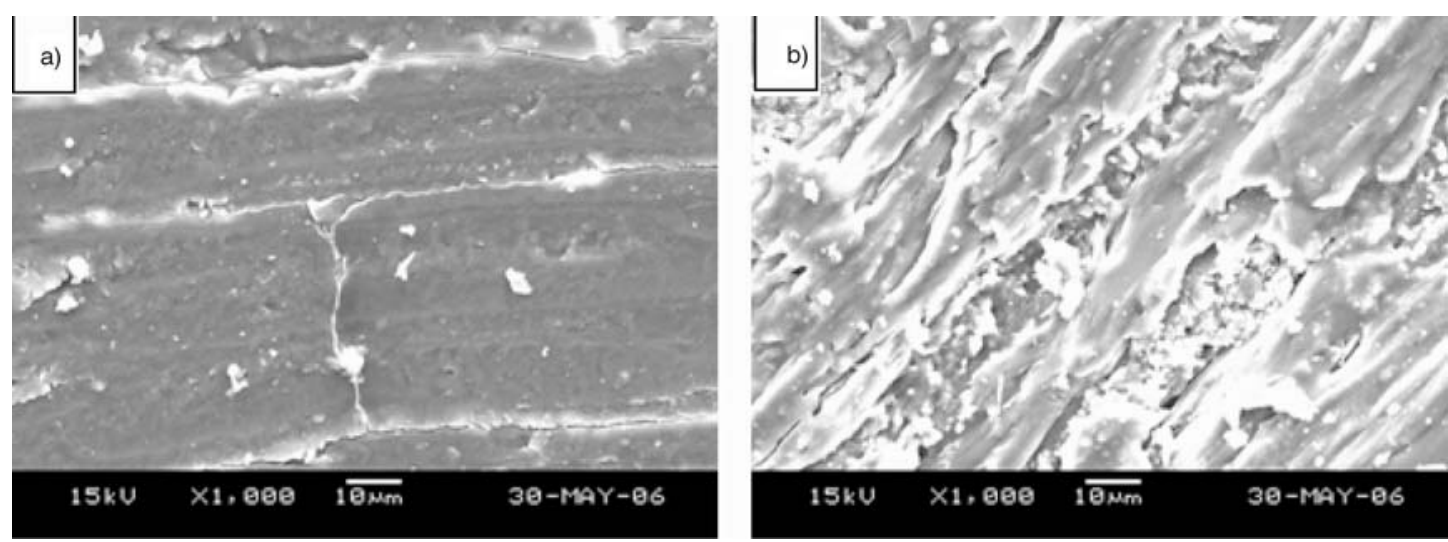

Figure 7. SEM micrograph of worn surface of SF/UFR composites with (a) $30 \mathrm{wt} \%$ and (b) $50 \mathrm{wt} \%$ sisal fibre

sliding and transfer to the interface between the composite and the steel counter-face. The thin transfer film adhering strongly to the metallic counterpart serves as spacer, preventing the direct contact between the two mating surfaces, thereby slowing the wear rate and reducing the friction coefficient. By contrast, as shown in Figure $7 b$, the worn surface of composite with $50 \mathrm{wt} \% \mathrm{SF}$ is quite rough, displaying cracks and microploughing furrows. Fibre and some other components are fractured into fragments and many small filler particles are detached from the composite leaving cavities in the surface. These cavities are themselves stress concentrators and resulted in more cracks and a higher friction coefficient.

\subsection{Water absorption property}

Figure 8 shows the result of water absorption. We can find out the water absorption of composite with $30 \mathrm{wt} \%$ SF is only $0.98 \mathrm{wt} \%$. This can be attributed to the greater adhesion between the fibre and matrix, the low water absorption nature of the matrix. It is observed that with increase of SF content from 30 to $70 \mathrm{wt} \%$, the water absorption rockets. This might be due to the poor distribution of fibres in the composite which result in the formation of fibre lumps. These lumps require more force to deform during hot pressing, and as a result more stresses are built in the composites [20]. When the composites are tested (water absorption test), a greater proportion of these stresses are released, causing a high water absorption. In addition, sisal fibres are lignocellulosics and therefore hydrophilic in nature; sisal absorbs moisture readily.

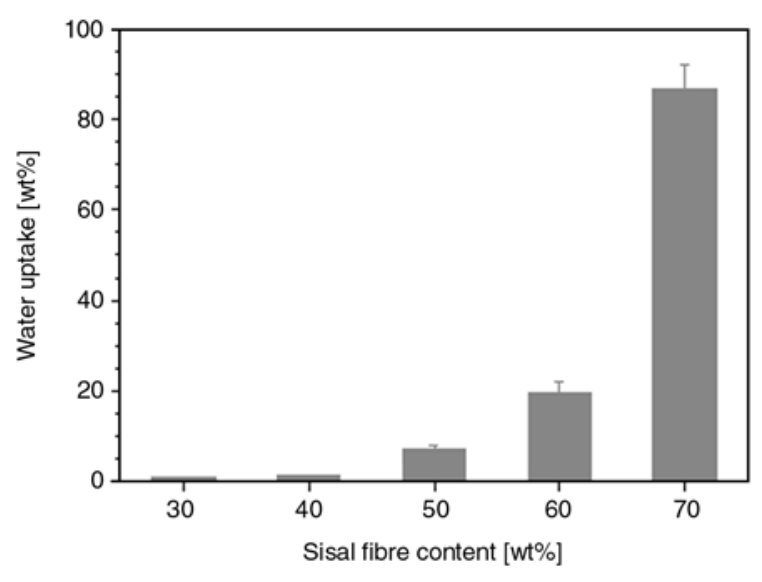

Figure 8. Effects of sisal fibre loading on the water absorption property of SF/UFR composites

\section{Conclusions}

In this paper, mechanical properties of SF reinforced urea-formaldehyde resin composites have been described. The composite with $50 \mathrm{wt} \%$ SF has the optimal Charpy impact strength and it reaches $9.42 \mathrm{~kJ} / \mathrm{m}^{2}$. Whereas the flexural, wear resistance and water absorption properties are proved to be excellent in the composite with $30 \mathrm{wt} \% \mathrm{SF}$ under the present experimental conditions adopted. The SEM micrographs of impact fractured and worn surfaces reveal that the proper sisal fibre addition can improve the fracture energy, strengthen interfaces and lower wear rate. The composite has significant potential for improving resistance to abrasive wear due to sisal characteristic properties. The fibres themselves possess a higher wear resistance than the matrix and should protrude from the surface after some time. Hence, the applicability of these composites in fibreboard can be expanded. 


\section{Acknowledgements}

The authors would like to acknowledge the Guangxi Science and Technology Bureau (Grant No.: 05112001-2A3) and Guangxi Natural Science Foundation (No.: 0728223) for financial support.

\section{References}

[1] Mohanty A. K., Misra M., Drzal L. T.: Surface modifications of natural fibers and performance of the resulting biocomposites: An overview. Composite Interfaces, 8, 313-343 (2001).

[2] Joseph K., Thomas S., Paul A.: Effect of surface treatments on the electrical properties of low-density polyethylene composites reinforced with short sisal fibers. Composites Science and Technology, 57, 67-79 (1997).

[3] Singh B., Gupta M., Verma A.: Polyester moulding compounds of natural fibres and wollastonite. Composites: Part A, 34, 1035-1043 (2003).

[4] Luyt A. S., Malunka M. E.: Composites of low-density polyethylene and short sisal fibres: the effect of wax addition and peroxide treatment on thermal properties. Thermochimica Acta, 426, 101-107 (2005).

[5] Kalaprasad G., Joseph K., Thomas S., Pavithran C.: Theoretical modelling of tensile properties of short sisal fibre-reinforced low-density polyethylene composites. Journal of Materials Science, 32, 4261-4267 (1997).

[6] Pal S., Mukhopadhayay D., Sanyal S., Mukherjea R.: Studies on process variables for natural fibre composites- effect of PEAP as interfacial agent. Journal of Applied Polymer Science, 35, 973-985 (1988).

[7] Bisanda E. T. N.: The effect of alkali treatment on the adhesion characteristics of sisal fibres. Applied Composite Materials, 7, 331-339 (2000).

[8] Joseph P. V., Joseph K., Thomas S.: Short sisal fiber reinforced polypropylene composites: the role of interface modification on ultimate properties. Composite Interfaces, 9, 171-205 (2002).

[9] Fung K. L., Xing X. S., Li R. K. Y., Tjong S. C., Mai Y-W.: An investigation on the processing of sisal fibre reinforced polypropylene composites. Composites Science and Technology, 63, 1255-1258 (2003).

[10] Wei C., Mu Q-H., Luo W-H.: Influences of treatment methods for sisal stems on properties of sisal fiber/formaldehyde resin composites. China Plastics, 17, 44-47 (2003).
[11] Niu Y-L., Zhong J-B., Yang X-W., Yu X-B., Wei C.: Effect of sisal fiber contents on wear resistance of sisal fiber/phenol formaldehyde resin in-situ composites. in 'Proceedings of 2005 International Conference on Advanced Fibers and Polymer Materials, Shanghai, China', 411-414 (2005).

[12] Zhong J-B., Niu Y-L., Lü J., Wei C.: Effect of steam explosion treatment on mechanical properties of sisal fiber/phenol-formaldehyde resin composite. China Plastics Industry, 34, 53-55 (2006).

[13] de Rodriguez N. L. G., Thielemans W., Dufresne A.: Sisal cellulose whiskers reinforced polyvinyl acetate nanocomposites. Cellulose, 13, 261-270 (2006).

[14] Alvarez V., Vazquez A., Bernal C.: Effect of microstructure on the tensile and fracture properties of sisal fiber/starch-based composites. Journal of Composite Materials, 40, 21-35 (2006).

[15] Gillah P. R., lrle M. A., Amartey S. A.: Development and production of laboratory scale novel MDF panels from composite and nonwoven mattresses of sisal and wood fibre mixtures. Holz als Roh- und Werkstoff, $\mathbf{5 8}$ 324-330 (2000).

[16] Manikandan Nair K. C., Thomas S., Groeninckx G.: Thermal and dynamic mechanical analysis of polystyrene composites reinforced with short sisal fibres. Composites Science and Technology, 61, 2519-2529 (2001).

[17] Li Y.: The investigation of fracture properties of sisal textile reinforced polymers. Acta Mechanica Solida Sinica, 17, 95-103 (2004).

[18] Mishra S., Mohanty A. K., Drzal L. T., Misra M., Parija S., Nayak S. K., Tripathy S. S.: Studies on mechanical performance of biofibre/glass reinforced polyester hybrid composites. Composites Science and Technology, 63, 1377-1385 (2003).

[19] van den Oever M. J. A., Bos H. L., Molenveld K.: Flax physical fibre structure and its effect on composite properties: impact strength and thermo-mechanical properties. Die Angewandte Makromolekulare Chemie, 272, 71-76 (1999).

[20] Krzysik A. M., Youngquist J. A., Myers G. E., Chahyadi I. S., Kolosick P. C.: Wood-polymer bonding in extruded and nonwoven web composite panels. in 'Proceedings of Wood Adhesives 1990, Madison, USA', 183-189 (1990). 